\title{
Comment on Table 1 of Farshad and Gerber: Reverse total shoulder arthroplasty - from the most to the least common complication
}

\author{
Mazda Farshad • Christian Gerber
}

Received: 7 November 2010 /Revised: 7 November 2010/Accepted: 8 November 2010/Published online: 11 January 2011

(C) Springer-Verlag 2011

Dear Editor,

We would like to thank Dr. Jonathan Levy who, when reviewing the online-first published article "Reverse total shoulder arthroplasty - from the most to the least common complication" [1] in International Orthopaedics, kindly alerted us to two errors in Table 1. Unfortunately, during the review of the manuscript we had overlooked errors in Table 1, which need correction. Although the content and message of the manuscript are not affected by the typographical errors we would like to outline two misprints. First, in the row "Levy et al. [48], 2007" there is a typographical error of 11 instead of 1 patient with a neurological complication. This complication resolved without additional treatment. The second misprint occurs in the row "Levy et al. [10], 2007" where scapular notching is reported in all instead of none of the patients. During preparation of this table, it was challenging to summarise the data reported in each of the articles in one table as a result of heterogeneity of reporting and differences in definitions. A corrected table is provided (Table 1) and should replace the original Table 1 for the reader of this article.

We regret these errors, apologise to the readers and are grateful to Dr. Levy whose attention has allowed us a timely correction of a much-regretted oversight.

Table 1 Complications of reverse total shoulder arthroplasty (RTSA) found in the literature (corrected)

\begin{tabular}{|c|c|c|c|c|c|c|c|c|c|c|c|}
\hline Study & $\begin{array}{l}\text { Patient } \\
\text { number }\end{array}$ & $\begin{array}{l}\text { Mean } \\
\text { follow-up } \\
\text { (months) }\end{array}$ & Hematoma & Infections & Instability & $\begin{array}{l}\text { Scapular } \\
\text { notching }\end{array}$ & $\begin{array}{l}\text { Glenoidal } \\
\text { complication }\end{array}$ & $\begin{array}{l}\text { Humeral } \\
\text { complications }\end{array}$ & $\begin{array}{l}\text { Fracture of } \\
\text { the acromion }\end{array}$ & $\begin{array}{l}\text { Neurological } \\
\text { complications }\end{array}$ & Prosthesis \\
\hline $\begin{array}{l}\text { Gilbart and Gerber, } \\
\text { unpublished data }\end{array}$ & 111 & 26 & $17(15 \%)$ & $1(1 \%)$ & $7(6 \%)$ & $21(19 \%)$ & $5(5 \%)$ & $6(5 \%)$ & & $4(4 \%)$ & Delta III \\
\hline Molé and Favard [1], 2006 & 527 & & $14(3 \%)$ & $27(5 \%)$ & $18(3.4 \%)$ & & $27(5 \%)$ & $11(2 \%)$ & $16(3 \%)$ & $6(1 \%)$ & Mostly Delta \\
\hline $\begin{array}{l}\text { Gerber et al., unpublished } \\
\text { data (series 2005-2009) }\end{array}$ & 230 & 22 & $5(2 \%)$ & $2(1 \%)$ & $4(2 \%)$ & $115(50 \%)$ & $7(3 \%)$ & $1(0.4 \%)$ & $5(2 \%)$ & $11(5 \%)$ & $\begin{array}{l}\text { Anatomical } \\
\text { Inverse }\end{array}$ \\
\hline Levy et al. [48], 2007 & 29 & 35 & & $1(3 \%)$ & $4(14 \%)$ & & $1(3 \%)$ & $1(3 \%)$ & & $1(3 \%)$ & Encore \\
\hline Levy et al. [10], 2007 & 19 & 44 & $1(5 \%)$ & $1(5 \%)$ & & $0(0 \%)$ & $2(10 \%)$ & $2(10 \%)$ & & & Encore \\
\hline Guery et al. [50], 2006 & 75 & 70 & & $3(4 \%)$ & $2(3 \%)$ & & $3(4 \%)$ & & & & Delta III \\
\hline Werner et al. [13], 2005 & 58 & 38 & $12(21 \%)$ & $2(3 \%)$ & $5(9 \%)$ & $56(96 \%)$ & $3(5 \%)$ & $1(2 \%)$ & $4(7 \%)$ & & Delta III \\
\hline Boileau et al. [4], 2005 & 45 & 40 & & $2(4 \%)$ & & $24(53 \%)$ & $1(2 \%)$ & $3(7 \%)$ & & & Delta III \\
\hline Frankle et al. [51], 2005 & 60 & 33 & & $2(3 \%)$ & & $0(0 \%)$ & $6(10 \%)$ & $1(2 \%)$ & $2(3 \%)$ & & $\begin{array}{l}\text { Reverse Shoulder } \\
\text { System }\end{array}$ \\
\hline Klein et al. [52], 2008 & 20 & 33 & & $2(10 \%)$ & $1(5 \%)$ & $1(5 \%)$ & $0(0 \%)$ & $0(0 \%)$ & & $0(0 \%)$ & Delta III \\
\hline Grassi et al. [15], 2009 & 23 & 42 & & $(0 \%)$ & $1(4 \%)$ & $16(70 \%)$ & $2(9 \%)$ & & $0(0 \%)$ & & Delta III \\
\hline Sirveaux et al. [14], 2004 & 80 & 45 & & $1(1 \%)$ & & $49(61 \%)$ & $12(15 \%)$ & & & & Delta III \\
\hline
\end{tabular}

\section{Reference}

M. Farshad $(\bowtie) \cdot$ C. Gerber

Balgrist University Hospital, University of Zürich,

Zürich, Switzerland

e-mail: mazda.farshad@balgrist.ch
1. Farshad M, Gerber C (2010) Reverse total shoulder arthroplastyfrom the most to the least common complication. Int Orthop. doi:10.1007/s00264-010-1125-2 